\title{
Combining computer adaptive testing technology with cognitively diagnostic assessment
}

\author{
Meghan McGlohen \\ University of Texas, Austin, Texas \\ AND \\ Hua-Hua Chang \\ University of Illinois at Urbana-Champaign, Urbana, Illinois
}

\begin{abstract}
A major advantage of computerized adaptive testing (CAT) is that it allows the test to home in on an examinee's ability level in an interactive manner. The aim of the new area of cognitive diagnosis is to provide information about specific content areas in which an examinee needs help. The goal of this study was to combine the benefit of specific feedback from cognitively diagnostic assessment with the advantages of CAT. In this study, three approaches to combining these were investigated: (1) item selection based on the traditional ability level estimate $(\theta),(2)$ item selection based on the attribute mastery feedback provided by cognitively diagnostic assessment $(\alpha)$, and (3) item selection based on both the traditional ability level estimate $(\theta)$ and the attribute mastery feedback provided by cognitively diagnostic assessment $(\alpha)$. The results from these three approaches were compared for $\theta$ estimation accuracy, attribute mastery estimation accuracy, and item exposure control. The $\theta$ - and $\alpha$-based condition outperformed the $\alpha$-based condition regarding $\theta$ estimation, attribute mastery pattern estimation, and item exposure control. Both the $\theta$-based condition and the $\theta$ - and $\alpha$-based condition performed similarly with regard to $\theta$ estimation, attribute mastery estimation, and item exposure control, but the $\theta$ - and $\alpha$-based condition has an additional advantage in that it uses the shadow test method, which allows the administrator to incorporate additional constraints in the item selection process, such as content balancing, item type constraints, and so forth, and also to select items on the basis of both the current $\theta$ and $\alpha$ estimates, which can be built on top of existing 3PL testing programs.
\end{abstract}

The goal of computerized adaptive testing (CAT) is to tailor a test to each individual examinee. CAT maintains many advantages in an assessment situation because it allows the test to home in on the examinee's ability level in an interactive manner. However, although traditional tests can accomplish assessment goals, such as a ranked comparison of examinees or grade assignments on the basis of certain criteria, they do not provide individualized information to teachers or test-takers regarding specific content in the domain of interest (Chipman, Nichols, \& Brennan, 1995). A new approach to educational research has begun to effloresce in order to address this issue. The aim of this research area, dealing with the application of cognitive diagnosis in the assessment process, is to provide helpful information to parents, teachers, and students, which can be used to direct additional instruction and study to the areas needed most by the individual student.

Current approaches whose interest is cognitive diagnosis focus solely on the estimation of the knowledge state, or attribute vector. This study proposes the combination of the estimation of individual ability levels $\left(\hat{\theta}_{j}\right)$ with an emphasis on the diagnostic feedback provided by individual attribute vectors $\left(\underline{\hat{\alpha}}_{j}\right)$, thus linking the current standard in testing technology with a new area of research whose aim is to help teachers and students benefit from the testing process.

By combining the advantages of CAT with the helpful feedback provided by cognitively diagnostic assessment, this study proposes a method for customized diagnostic testing. The technique utilizes the shadow-testing algorithm to simultaneously optimize the estimation of both the ability level $\hat{\theta}_{j}$ and the attribute vector $\underline{\hat{\hat{\alpha}}}_{j}$.

By combining the traditional IRT-based CAT model with a cognitive diagnosis model, examinees will be able to obtain both an ability level estimator and feedback concerning their states on the measured attributes, instead of just one or the other.

\section{LITERATURE REVIEW}

\section{Cognitively Diagnostic Assessment}

Several models have been proposed to provide cognitively diagnostic information in the assessment process. Two of the founding models for cognitive diagnosis are

M. McGlohen, meghan.mcglohen@austin.utexas.edu 
Fischer's (1973) linear logistic trait model and Tatsuoka and Tatsuoka's (1982) rule space methodology. A plethora of additional models have used the concepts of these methods as foundations for new approaches or applications (for details, see Hartz, Roussos, \& Stout, 2002). Specifically, the unified model, developed by DiBello, Stout, and Roussos (1995), is based on the rule space model, and in turn, the fusion model (Hartz et al., 2002) is based on the unified model. The item response function for the fusion model is illustrated below (in Equation 1), as described by Hartz et al.:

$$
P\left(X_{i j}=1 \mid \underline{\alpha}_{j}, \eta_{j}\right)=\pi_{i}^{*} \prod_{k=1}^{K} r_{i k}^{*\left(-\alpha_{j k}\right) \times q_{i k}} P_{c_{i}}\left(\eta_{j}\right),
$$

where

$P_{c_{i}}\left(\eta_{j}\right)=$ the Rasch model with difficulty parameter $c_{i}$, $\pi_{i}^{*}=$ the probability of correctly applying all item $i$ required attributes given that the individual processes all of the required attributes for the item $i$,

$r_{i k}^{*}=\frac{P\left(Y_{i j k}=1 \mid \alpha_{j k}=0\right)}{P\left(Y_{i j k}=1 \mid \alpha_{j k}=1\right)}$, considered an attribute-based

item discrimination parameter for item $i$ and attribute $k$,

$Y_{j k}=1$ when examinee $j$ correctly applies attribute $k$ to item $i$, and 0 otherwise, and

$\alpha_{j k}=1$ when examinee $j$ has mastered attribute $k$, and 0 otherwise.

Also, the attribute vector for individual $j$ is denoted as $\underline{\alpha}_{j}$, and $\eta_{j}$ is the residual ability parameter, which deals with content measured by the test that is not included in the $Q$-matrix. (Please note that sometimes the notation of $\theta_{j}$ has been used to denote this residual ability; $\eta_{j}$ is used here to distinguish this parameter from the single ability score $\theta_{j}$ of the three-parameter logistic [3PL] model.)

A software program called Arpeggio (Educational Testing Service, 2004) is available as a means of analyzing examinee response data to provide diagnostic feedback in the form of individual attribute vectors. The next helpful step in the diagnostic process would be to implement CAT technologies to optimize the item selection process with respect to the estimation process.

\section{Shadow Testing by Means \\ of Integer Programming}

Shadow testing, a mode for test assembly that utilizes integer programming (IP) to incorporate constraints into the assembly process, was proposed by van der Linden and Reese (1998). It is an iterative process in which an ideal "shadow" test is formed before the administration of each item in an examination. Each shadow test must contain items already included in the test and must be optimal at the given estimate level, while complying with all of the specified constraints (van der Linden \& Chang, 2003). The best item on the shadow test is then selected as the next item to be administered. Then the response from this item is used in the process of formulating the next shadow test. The application of this approach results in two major advantages. First, the items actually administered in the adaptive test will follow the constraints, because each of the shadow tests meets these specifications. Second, the adaptive test will converge optimally to the true value of the estimator, because the shadow tests are assembled to be optimal for the current estimate level and, in turn, each selected item is the optimal one from that shadow test (van der Linden \& Chang, 2003).

Once a shadow test is constructed, the best item with respect to the attribute vector estimate is selected to be the next item administered to the examinee. Two strategies, Shannon entropy and Kullback-Leibler (K-L) information, are employed for the selection process, as described in Xu, Chang, and Douglas (2003).

Shannon entropy. Shannon entropy was developed in 1948 as a measure of uncertainty associated with a random variable. It is a nonnegative concave function of the probability distribution. In this context, Shannon entropy is minimized; that is to say, it is desirable to have minimal uncertainty. Shannon entropy is described by

$$
\operatorname{Sh}(\underline{\pi})=\sum_{i=1}^{K} \pi_{i} \log \left(\frac{1}{\pi_{i}}\right),
$$

where $\pi_{i}$ is the probability that the random variable of interest, call it $Y$, will take on a particular value $y_{i}$, and $\underline{\pi}$ is the probability vector containing the $\pi_{i}$ s for all possible values of $y_{i}$ (Xu et al., 2003). In the context of diagnostic assessment, where we are interested in estimating attribute vectors, the function for Shannon entropy becomes Equation 3, as described by Xu et al.:

$$
\begin{aligned}
\operatorname{Sh}\left(\underline{\pi_{n}}, X_{j}\right)= & \sum_{x=0}^{1} E_{n}\left(\underline{\pi_{n}} \mid X_{j}=x\right) P\left[X_{j}=x \mid \underline{\pi_{n-1}}\right] \\
= & \sum_{x=0}^{1}\left\{E_{n}\left(\underline{\pi_{n}} \mid X_{j}=x\right)\right. \\
& \left.\cdot\left(\sum_{c=1}^{2^{M}} P_{j}^{x}\left(\underline{\alpha_{c}}\right)\left[1-P_{j}\left(\underline{\alpha_{c}}\right)\right]^{-1-x} \pi_{n-1}\left(\underline{\alpha_{c}}\right)\right)\right\}
\end{aligned}
$$

Kullback-Leibler information. $\mathrm{K}-\mathrm{L}$ information was introduced in 1951 as a distance measure between probability distributions. More recently, $\mathrm{K}-\mathrm{L}$ information can be used as a measure of global information for the purpose of item selection in IRT (Chang \& Ying, 1999) and as an index in the item selection process in diagnostic assessment (Xu et al., 2003):

$$
K(f, g)=\int \log \left(\frac{f(x)}{g(x)}\right) f(x) \mu(d x) .
$$

For the cognitive diagnosis context, $\mathrm{K}-\mathrm{L}$ information is used as an item selection criterion. Since the variables are discrete, $K(f, g)$ is computed as a sum over all possible attribute patterns; then the sum is taken across all possible attribute patterns. Thus, the function becomes

$$
K_{j}(\underline{\hat{\alpha}})=\sum_{c=1}^{2^{M}}\left[\sum_{x=0}^{1} \log \left(\frac{P\left(X_{j}=x \mid \underline{\hat{\alpha}}\right)}{P\left(X_{j}=x \mid \underline{\alpha_{c}}\right)}\right) P\left(X_{j}=x \mid \underline{\hat{\alpha}}\right)\right] \text {, }
$$


where $\underline{\hat{\alpha}}$ is the current estimate for the attribute vector and $\alpha_{c}$ is the possible candidate attribute vector generated by the $j$ th item (Xu et al., 2003). This yields an information index relating our current attribute vector estimate to the possible attribute vector estimate resulting from the administration of the next item $(j)$ for every possible remaining item. Recall that the goal when Shannon entropy is used to minimize uncertainty, but when $\mathrm{K}-\mathrm{L}$ information is used, the goal becomes to maximize information. Thus, the item with the largest value of $K_{j}(\underline{\hat{\alpha}})$ is then selected as the next item.

In this study, maximizing Fischer information with respect to the current estimate of the single score, $\hat{\theta}_{j}$, was used as the optimization function of the LP in the shadow test. Then the minimization of Shannon entropy and the maximization of $\mathrm{K}-\mathrm{L}$ information with respect to the attribute mastery vector, $\underline{\hat{\alpha}}_{j}$, were used as different approaches to select the best item from the shadow test. Thus, the item selected to be administered next would be a good item with respect to both $\theta$ and attribute mastery estimation and would obey any specified constraints in the LP. Van der Linden and Reese (1998) outlined several nonstatistical constraints of interest, including content balance, item time availability, and test length. In this study, a similar approach was used to apply these nonstatistical constraints.

\section{METHOD}

The item parameters were precalibrated on the basis of a simple random sample of 2,000 examinees of three administrations of a state-mandated large-scale assessment, using Bilog MG and Arpeggio 1.2 to obtain the 3PL and fusion-model-based item parameters, respectively. This large-scale assessment consisted of a math portion and a reading portion. The item parameters from the three administrations were tripled to increase the size of the item bank. This resulted in item banks with 396 items for the math portion of the test and 324 items for the reading portion. The examinees' estimated parameters (both single score $\theta \mathrm{s}$ and attribute mastery patterns) were used as true scores in the CAT simulation. Out of the 6,000 examinees, 3,000 were randomly sampled with replacement to be employed in the CAT simulation.

This study's design included three conditions for comparison: one in which items were selected on the basis of the $\hat{\theta}$ estimate only, one in which items were selected on the basis of the $\underline{\hat{\alpha}}$ estimate only, and one in which items were selected on the basis of both the $\hat{\theta}$ and the $\underline{\hat{\alpha}}$ estimates. Within each of the three conditions, there were two different subconditions based on the algorithm used for item selection.

In the first condition, the conventional method of focusing solely on $\hat{\theta}$ was used during item selection and then the $\hat{\alpha}$ vectors were estimated at the end, using MLE estimation. This condition will be referred to as the $\theta$-based condition, because item selection was based on the $\hat{\theta}$ estimates only. Within this condition based only on the $\hat{\theta}$ estimates, two different algorithms were employed for selecting the next item on the test: one based on maximizing Fischer in- formation and the other based on maximizing $\mathrm{K}-\mathrm{L}$ information. For this condition, the probabilities of obtaining a correct response were computed using the 3PL model, because this condition was based on the $\hat{\theta}$ estimates only.

In the second condition, focusing solely on $\underline{\hat{\alpha}}$, the approach outlined in Xu et al. (2003) was mimicked to select items and the $\hat{\theta}$ values were calculated afterward on the basis of the response patterns, using MLE estimation. This condition will be referred to as the $\alpha$-based condition, because item selection was based on the $\hat{\alpha}$ estimates only. Within this condition, two different algorithms were employed for selecting the next item on the test: one based on minimizing Shannon entropy and the other based on maximizing $\mathrm{K}-\mathrm{L}$ information. For this condition, the probabilities of obtaining a correct response were computed using the fusion model, because this condition was based on the $\underline{\hat{\alpha}}$ estimates only.

The third condition was the heart of the study, and both the $\hat{\theta}$ and the $\hat{\alpha}$ estimates were used in combination to select the next item to be administered in the test. This condition will be referred to as the $\theta$ - and $\underline{\alpha}$-based condition, because item selection was based on both estimates. This condition first involved the construction of a shadow test that was optimized according to the ability level $\hat{\theta}$ (as outlined by van der Linden \& Reese, 1998) before the administration of each item. Then the best item for measuring the attribute vector $\underline{\hat{\alpha}}$ was selected from the shadow test on the basis of the current $\underline{\hat{\alpha}}$ estimate, using Shannon entropy or $\mathrm{K}-\mathrm{L}$ information as outlined in $\mathrm{Xu}$ et al. (2003). This was the only condition involving the shadow test approach. Note that the two subconditions based on the algorithm for selecting the next item were the same for the $\underline{\alpha}$-based condition and the $\theta$ - and $\underline{\alpha}$-based condition. This condition was applied to two scenarios: one in which the probabilities of obtaining a correct response were computed using the 3PL model and another in which the probabilities of obtaining a correct response were computed using the fusion model, because this condition was based on both the $\hat{\theta}$ and the $\underline{\hat{\alpha}}$ estimates. Hence, this condition needed to be compared with the other two conditions separately. Examining both of these approaches to computing the probabilities (3PL based and fusion based) should demonstrate the robustness of this method.

Lastly, an item exposure control method was selected. For the $\theta$-based condition and the $\theta$ - and $\alpha$-based condition, the 5-4-3-2-1 item exposure control method proposed by McBride and Martin (1983) was implemented. The results of the three different conditions were compared with regard to the accuracy of both the attribute classification rate and the ability levels by comparing the estimated values with the true values.

\section{RESULTS}

The evaluation of the information obtained from the various conditions involves three areas of examination. First, it is important that the various methods accurately estimated the values of the single score $(\theta)$. Second, the methods should also accurately estimate the attribute mastery patterns $(\underline{\alpha})$. An acceptable method would estimate 
both the $\theta$ values and the attribute mastery patterns well. Third, the item exposure rates of the various methods are examined, because item exposure control is an important issue in test security in computerized adaptive testing. The following sections will deal with each of these issues. Optimally, examinees' single scores and attribute vectors should be accurately estimated while maintaining minimal item exposure rates for test security.

When the accuracy of $\theta$ estimation and the correct attribute classification are compared, the three conditions are compared with each other in pairs on the basis of whether the individual response patterns were computed using the 3PL model or the fusion model. For one comparison, approaches in which the probability of obtaining a correct response is based on the 3PL model are grouped together. More specifically, the $\theta$-based condition and the $\hat{\theta}$ - and $\underline{\alpha}$-based condition are considered the 3PL-based approaches. Likewise, approaches in which the probabilities for obtaining a correct response are based on the fusion model are grouped together in the other comparison. This comparison of fusion-based approaches would be between the $\underline{\alpha}$-based condition and the $\theta$ - and $\underline{\alpha}$-based condition.

It would be impractical to compare results across these differences (i.e., comparing the 3PL-based approaches against the fusion-based approaches), because how the response patterns are generated is a fundamental aspect of the CAT simulation and it would be ineffective to distinguish the differences due to model choice from the differences due to the various methods and conditions. Note that the $\theta$ - and $\underline{\alpha}$-based condition is present in both the 3PL-based comparison and the fusion-based comparison, because the $\theta$ - and $\underline{\alpha}$-based condition was conducted utilizing both the 3PL model and the fusion model separately in the shadow-testing approach to item selection. This study utilized two different approaches to computing item response probabilities - 3PL based and fusion based - but it should be noted that the 3PL model (including the 1PL and 2PL subsets) is the one frequently used in real testing programs, making the 3PL-based comparison (between the $\theta$-based condition and the $\theta$ - and $\underline{\alpha}$-based condition) the more interesting comparison.

\section{$\theta$ Estimation}

First, the $\theta$ estimates of the different conditions are of particular interest. The $\theta$ estimates are compared with the true $\theta$ values to determine how well each of the methods succeeds in accurately estimating the single-score $\theta$. The comparison between the true $\theta$ and its corresponding estimate is accomplished by examining the values of the correlation coefficient, the root-mean square error, and the bias statistics.

Correlation coefficients for all of the item selection methods within each condition are presented in Table 1 for the response probabilities based on the 3PL model and in Table 2 for the response probabilities based on the fusion model. Values of bias are presented in Table 3 for probabilities based on the 3PL model and in Table 4 for those based on the fusion model. The root-mean square error values are presented in Table 5 for probabilities based on
Table 1

Correlations of the True $\theta$ Values and the Estimated $\theta$ Values for Three-Parameter-Logistic-Based Probabilities

\begin{tabular}{cccccc}
\hline & \multicolumn{2}{c}{$\begin{array}{c}\theta \text {-Based } \\
\text { Condition }\end{array}$} & & \multicolumn{2}{c}{$\begin{array}{c}\theta \text {-and } \underline{\alpha} \text {-Based } \\
\text { Condition }\end{array}$} \\
\cline { 2 - 3 }$Q$-Matrix & Fischer & K-L & & Shannon & K-L \\
\hline Math & & & & & \\
Blueprint & .965 & .966 & & .969 & .971 \\
Intuitive & .967 & .967 & & .975 & .975 \\
Reading & & & & & \\
Blueprint & .950 & .954 & & .960 & .956 \\
Intuitive & .952 & .950 & & .951 & .956
\end{tabular}

Note-K-L, Kullback-Leibler.

Table 2

Correlations of the True $\theta$ Values and the Estimated $\theta$ Values for Fusion-Based Probabilities

\begin{tabular}{|c|c|c|c|c|}
\hline \multirow[b]{2}{*}{$Q$-Matrix } & \multicolumn{2}{|c|}{$\underline{\alpha}$-Based Condition } & \multicolumn{2}{|c|}{$\begin{array}{l}\theta \text { - and } \underline{\alpha-B a s e d} \\
\text { Condition }\end{array}$} \\
\hline & Shannon & $\mathrm{K}-\mathrm{L}$ & Shannon & $\mathrm{K}-\mathrm{L}$ \\
\hline \multicolumn{5}{|l|}{ Math } \\
\hline Blueprint & .768 & .782 & .786 & .790 \\
\hline Intuitive & .795 & .749 & .817 & .812 \\
\hline \multicolumn{5}{|l|}{ Reading } \\
\hline Blueprint & .718 & .763 & .752 & .762 \\
\hline Intuitive & .203 & .222 & .227 & .230 \\
\hline
\end{tabular}

Note-K-L, Kullback-Leibler.

Table 3

Bias Statistics of the Estimated $\theta$ Values for Three-Parameter-Logistic-Based Probabilities

\begin{tabular}{|c|c|c|c|c|}
\hline \multirow[b]{2}{*}{$Q$-Matrix } & \multicolumn{2}{|c|}{$\theta$-Based Condition } & \multicolumn{2}{|c|}{$\begin{array}{l}\theta-\text { and } \underline{\alpha-B a s e d} \\
\text { Condition }\end{array}$} \\
\hline & Fischer & $\mathrm{K}-\mathrm{L}$ & Shannon & $\mathrm{K}-\mathrm{L}$ \\
\hline \multicolumn{5}{|l|}{ Math } \\
\hline Blueprint & -.031 & -.036 & -.022 & -.023 \\
\hline Intuitive & -.019 & -.008 & -.017 & -.011 \\
\hline \multicolumn{5}{|l|}{ Reading } \\
\hline Blueprint & -.062 & -.057 & -.047 & -.050 \\
\hline Intuitive & -.049 & -.060 & -.055 & -.054 \\
\hline
\end{tabular}

Note-K-L, Kullback-Leibler.

Table 4

Bias Statistics of the Estimated $\theta$ Values for Fusion-Based Probabilities

\begin{tabular}{cccccr}
\hline & \multicolumn{2}{c}{$\begin{array}{c}\alpha \text {-Based } \\
\text { Condition }\end{array}$} & & \multicolumn{2}{c}{$\begin{array}{c}\text { and } \underline{\alpha} \text {-Based } \\
\text { Condition }\end{array}$} \\
\cline { 2 - 3 } \cline { 5 - 6 }$Q$-Matrix & Shannon & $\mathrm{K}-\mathrm{L}$ & & Shannon & $\mathrm{K}-\mathrm{L}$ \\
\hline Math & & & & & \\
Blueprint & .103 & .164 & & .093 & .073 \\
Intuitive & .077 & .069 & & .078 & .097 \\
Reading & & & & & .081 \\
Blueprint & .160 & .144 & & .061 & \\
Intuitive & .674 & .113 & -.190 & -.426 \\
\hline
\end{tabular}

Note-K-L, Kullback-Leibler. 
the 3PL model and in Table 6 for probabilities based on the fusion model.

First, the results from the 3PL-based simulations will be examined. In Table 1, all the correlations are above .9, and the values are similar across the $\theta$-based condition and the $\theta$ - and $\underline{\alpha}$-based condition. Correlations are typically lower for the reading test than for the math test. Similarly, all of the bias values in Table 3 are small and similar across the two conditions but are smaller for the math test than for the reading test. The root-mean square errors also tend to be greater for the reading test. Note that, in general, the methods perform more poorly on the reading test than on the math test. Overall, the math test seems to have more accurate estimates than does the reading test, which indicates that the reading test is not as good at measuring a single overall reading score as the math test is at measuring a single overall math score.

In Table 5, the root-mean square error is lower in the $\theta$-based condition for the math test but is lower for the $\theta$ - and $\underline{\alpha}$-based condition in the reading test. Overall, for the 3PL approach to calculating response probabilities, the $\theta$-based condition and the $\theta$ - and $\underline{\alpha-b a s e d ~ c o n d i t i o n ~ s e e m ~}$ to have comparable performances at accurately measuring the single-score $\theta$ for the examinees when the 3PL model is used for calculating response probabilities. Within the $\theta$-based condition, item selection based on maximizing Fischer information and that based on $\mathrm{K}-\mathrm{L}$ information tend to perform equally well, as do minimizing Shannon entropy and maximizing $\mathrm{K}-\mathrm{L}$ information within the $\theta$ - and $\underline{\alpha}$-based condition.

Next, the results of the methods using the fusion model to calculate response probabilities are examined. As was expected, conditions in which the probabilities of obtaining a correct response are based on the fusion model do not estimate the values of the single-score $\theta$ very well, as depicted by the correlations of true $\theta$ values and estimated $\theta$ values ranging from .203 to .817 in Table 2, with the lowest correlations computed for the reading test that used the intuitive $Q$-matrix. This illustrates the importance of $Q$-matrix construction when implementing the fusion model.

Note that poor estimations of $\theta$ were predicted for the condition that used fusion-based probabilities, because the value of $\theta$ is not present anywhere in the probability function for the item responses. So, implicitly, the algorithm will not perform well at estimating a parameter that is not used in determining whether the response to each item is correct or incorrect. Hence, the values of the correlation coefficients are lower and the root-mean square error and bias values are greater than desired, but what is more interesting for this study is a comparison between the different methods within this fusion-based model approach. Within the fusion-based probabilities, higher correlations, lower bias estimates, and lower root-mean square error values in Tables 2, 4, and 6, respectively, illustrate that the $\theta$ - and $\underline{\alpha}$-based condition performs better than the $\underline{\alpha}$-based condition at estimating single $\theta$ scores. This means that an item selection method that takes $\theta$ estimates and attribute mastery patterns into account yields better $\theta$ estimates than does the item selection method
Table 5

Root-Mean Square Errors of the Estimated $\boldsymbol{\theta}$ Values for Three-Parameter-Logistic-Based Probabilities

\begin{tabular}{cccccc}
\hline & \multicolumn{2}{c}{$\begin{array}{c}\theta \text {-Based } \\
\text { Condition }\end{array}$} & & \multicolumn{2}{c}{$\begin{array}{c}\theta \text { and } \underline{\alpha-B a s e d} \\
\text { Condition }\end{array}$} \\
\cline { 2 - 3 }$Q$-Matrix & Fischer & $\mathrm{K}-\mathrm{L}$ & & Shannon & $\mathrm{K}-\mathrm{L}$ \\
\hline Math & & & & & \\
Blueprint & 0.296 & 0.298 & & 0.282 & 0.265 \\
Intuitive & 0.295 & 0.293 & & 0.262 & 0.258 \\
Reading & & & & & \\
Blueprint & 0.335 & 0.324 & & 0.302 & 0.318 \\
Intuitive & 0.324 & 0.334 & & 0.329 & 0.317 \\
\hline
\end{tabular}

Note-K-L, Kullback-Leibler.

Table 6

Root-Mean Square Errors of the Estimated $\boldsymbol{\theta}$ Values for Fusion-Based Probabilities

\begin{tabular}{|c|c|c|c|c|}
\hline \multirow[b]{2}{*}{$Q$-Matrix } & \multicolumn{2}{|c|}{$\underline{\alpha}$-Based Condition } & \multicolumn{2}{|c|}{$\begin{array}{l}\theta-\text { and } \underline{\alpha-B a s e d} \\
\text { Condition }\end{array}$} \\
\hline & Shannon & $\mathrm{K}-\mathrm{L}$ & Shannon & $\mathrm{K}-\mathrm{L}$ \\
\hline \multicolumn{5}{|l|}{ Math } \\
\hline Blueprint & 0.692 & 0.685 & 0.674 & 0.666 \\
\hline Intuitive & 0.689 & 0.748 & 0.658 & 0.667 \\
\hline \multicolumn{5}{|l|}{ Reading } \\
\hline Blueprint & 0.748 & 0.681 & 0.696 & 0.687 \\
\hline Intuitive & 1.351 & 1.174 & 1.364 & 1.345 \\
\hline
\end{tabular}

Note-K-L, Kullback-Leibler.

Table 7

Math Test's Correct Attribute Mastery Classifications (Hit Rates) Using Three-Parameter-Logistic-Based Probabilities

\begin{tabular}{|c|c|c|c|c|c|}
\hline \multirow[b]{2}{*}{$Q$-Matrix } & \multirow[b]{2}{*}{ Attribute } & \multicolumn{2}{|c|}{$\begin{array}{c}\theta \text {-Based } \\
\text { Condition }\end{array}$} & \multicolumn{2}{|c|}{$\begin{array}{l}\theta-\text { and } \underline{\alpha} \text {-Based } \\
\text { Condition }\end{array}$} \\
\hline & & Fischer & $\mathrm{K}-\mathrm{L}$ & Shannon & $\mathrm{K}-\mathrm{L}$ \\
\hline \multirow[t]{13}{*}{ Blueprint } & 1 & .797 & .792 & .789 & .795 \\
\hline & 2 & .712 & .696 & .715 & .703 \\
\hline & 3 & .686 & .681 & .678 & .710 \\
\hline & 4 & .710 & .725 & .718 & .703 \\
\hline & 5 & .783 & .796 & .780 & .794 \\
\hline & 6 & .833 & .827 & .833 & .816 \\
\hline & 7 & .808 & .810 & .805 & .816 \\
\hline & 8 & .814 & .817 & .808 & .818 \\
\hline & 9 & .557 & .564 & .585 & .556 \\
\hline & 10 & .807 & .796 & .825 & .823 \\
\hline & 11 & .746 & .748 & .763 & .770 \\
\hline & Mean 1-11 & .750 & .750 & .754 & .755 \\
\hline & Whole pattern & .169 & .162 & .169 & .176 \\
\hline \multirow[t]{15}{*}{ Intuitive } & 1 & .835 & .838 & .863 & .838 \\
\hline & 2 & .813 & .823 & .804 & .746 \\
\hline & 3 & .826 & .824 & .834 & .833 \\
\hline & 4 & .765 & .765 & .706 & .720 \\
\hline & 5 & .795 & .806 & .772 & .779 \\
\hline & 6 & .801 & .764 & .797 & .804 \\
\hline & 7 & .741 & .749 & .711 & .720 \\
\hline & 8 & .804 & .809 & .778 & .757 \\
\hline & 9 & .800 & .813 & .848 & .828 \\
\hline & 10 & .622 & .598 & .672 & .659 \\
\hline & 11 & .847 & .860 & .790 & .776 \\
\hline & 12 & .825 & .822 & .767 & .758 \\
\hline & 13 & .685 & .664 & .646 & .622 \\
\hline & Mean 1-13 & .782 & .780 & .768 & .757 \\
\hline & Whole pattern & .220 & .211 & .206 & .193 \\
\hline
\end{tabular}

Note-K-L, Kullback-Leibler. 
Table 8

Reading Test's Correct Attribute Mastery Classifications (Hit Rates) Using Three-Parameter-Logistic-Based Probabilities

\begin{tabular}{|c|c|c|c|c|c|}
\hline \multirow[b]{2}{*}{$Q$-Matrix } & \multirow[b]{2}{*}{ Attribute } & \multicolumn{2}{|c|}{$\begin{array}{c}\theta \text {-Based } \\
\text { Condition }\end{array}$} & \multicolumn{2}{|c|}{$\begin{array}{l}\theta-\text { and } \alpha \text {-Based } \\
\text { Condition }\end{array}$} \\
\hline & & Fischer & $\mathrm{K}-\mathrm{L}$ & Shannon & $\mathrm{K}-\mathrm{L}$ \\
\hline \multirow[t]{8}{*}{ Blueprint } & 1 & .830 & .830 & .787 & .781 \\
\hline & 2 & .847 & .850 & .868 & .863 \\
\hline & 3 & .795 & .779 & .797 & .802 \\
\hline & 4 & .863 & .861 & .856 & .864 \\
\hline & 5 & .815 & .824 & .829 & .823 \\
\hline & 6 & .827 & .834 & .843 & .861 \\
\hline & Mean 1-6 & .829 & .830 & .830 & .832 \\
\hline & Whole pattern & .586 & .590 & .583 & .580 \\
\hline \multirow[t]{9}{*}{ Intuitive } & 1 & .758 & .758 & .756 & .754 \\
\hline & 2 & .814 & .812 & .799 & .808 \\
\hline & 3 & .751 & .760 & .753 & .723 \\
\hline & 4 & .753 & .744 & .767 & .754 \\
\hline & 5 & .787 & .787 & .784 & .792 \\
\hline & 6 & .766 & .767 & .762 & .769 \\
\hline & 7 & .792 & .788 & .804 & .803 \\
\hline & Mean 1-7 & .774 & .774 & .775 & .772 \\
\hline & Whole pattern & .468 & .465 & .465 & .443 \\
\hline
\end{tabular}

Note-K-L, Kullback-Leibler.

that takes only attribute mastery patterns into account. To evaluate which method(s) are best overall, however, the accuracy of the attribute mastery classifications must also be considered.

\section{Attribute Mastery Estimation}

Optimally, an assessment approach will accurately estimate the attribute mastery of each attribute individually, as well as the entire attribute pattern overall for the examinees. To evaluate the attribute mastery estimation, the correct classification rates of each measured attribute and the entire attribute pattern are presented in the following tables. Table 7 presents these correct classification rates, or hit rates, of each method, using the 3PL to determine the response pattern probabilities for the math test. Table 8 holds the same information for the reading test. Tables 9 and 10 present the attributes' correct classification rates for the fusion-model-based probabilities for the math test and the reading test, respectively. A list of the attributes measured by each $Q$-matrix for each subject portion is presented in the Appendix.

This information may be more easily compared across the various approaches through graphical representation. The correct classification rates of the attribute mastery estimates are illustrated graphically in Figures 1-8.

With regard to the approaches based on the 3PL model for determining item response probabilities, the $\theta$-based condition and the $\theta$ - and $\underline{\alpha}$-based condition for the math and reading portions of the test on the individual attribute level, as well as for the entire attribute pattern, an examination of these figures indicates that within the $\theta$-based condition, both the Fischer information and $\mathrm{K}-\mathrm{L}$ information perform equally well in selecting items on the basis of the current $\theta$ estimate.

Results are more irregular for the methods in which the item response probabilities were based on the fusion model. Note that for the math test, the $\underline{\alpha}$-based condition and the $\theta$ - and $\underline{\alpha}$-based condition performed similarly, but a holistic examination shows that the $\theta$ - and $\underline{\alpha}$-based condition classified the examinees as masters or nonmasters of the attributes more consistently, whereas the $\underline{\alpha}$-based condition showed more fluctuation. Within the $\underline{\alpha}$-based condition, using Shannon entropy seems to have produced more accurate attribute mastery classifications than did using $\mathrm{K}-\mathrm{L}$ information. For the reading portion, the second condition yielded slightly higher correct classifications for many of the attributes and for the overall mastery patterns, and overall, the differences between the two condition's correct classification rates are quite small. It is surprising that performance in the $\underline{\alpha}$-based condition was not much better than that in the $\theta$ - and $\underline{\alpha}$-based condition. In the $\underline{\alpha}$-based condition, items were selected only on the basis of the current attribute mastery pattern estimate, $\underline{\alpha}_{j}$, and in the $\theta$ - and $\underline{\alpha}$-based condition, both $\underline{\alpha}_{j}$ and $\theta_{j}$ were taken into account, so it is logical that performance in the $\underline{\alpha}$-based condition should be quite a bit better than that in the $\theta$ - and $\underline{\alpha}$-based condition with regard to correct attribute mastery estimation; but in actuality, the results were comparable across the two conditions. As a result, a test administrator would not have to sacrifice much attribute mastery classification precision in order to obtain the higher precision in $\theta$ estimates found in the $\theta$ - and $\underline{\alpha}$-based condition. (Recall

Table 9

Math Test's Correct Attribute Mastery Classifications (Hit Rates) Using Fusion-Based Probabilities

\begin{tabular}{|c|c|c|c|c|c|}
\hline \multirow[b]{2}{*}{$Q$-Matrix } & \multirow[b]{2}{*}{ Attribute } & \multicolumn{2}{|c|}{$\begin{array}{l}\underline{\alpha} \text {-Based } \\
\text { Condition }\end{array}$} & \multicolumn{2}{|c|}{$\begin{array}{l}\theta \text { - and } \underline{\alpha} \text {-Based } \\
\text { Condition }\end{array}$} \\
\hline & & Shannon & $\mathrm{K}-\mathrm{L}$ & Shannon & $\mathrm{K}-\mathrm{L}$ \\
\hline \multirow[t]{13}{*}{ Blueprint } & 1 & .233 & .205 & .797 & .801 \\
\hline & 2 & .849 & .871 & .800 & .801 \\
\hline & 3 & .847 & .222 & .688 & .703 \\
\hline & 4 & .887 & .847 & .781 & .797 \\
\hline & 5 & .274 & .853 & .882 & .878 \\
\hline & 6 & .356 & .877 & .890 & .876 \\
\hline & 7 & .894 & .904 & .879 & .898 \\
\hline & 8 & .816 & .907 & .883 & .885 \\
\hline & 9 & .882 & .368 & .643 & .554 \\
\hline & 10 & .939 & .997 & .937 & .939 \\
\hline & 11 & .907 & .955 & .813 & .835 \\
\hline & Mean 1-11 & .717 & .728 & .817 & .815 \\
\hline & Whole pattern & .040 & .007 & .160 & .170 \\
\hline \multirow[t]{15}{*}{ Intuitive } & 1 & .905 & .242 & .882 & .855 \\
\hline & 2 & .802 & .159 & .786 & .736 \\
\hline & 3 & .925 & .876 & .896 & .876 \\
\hline & 4 & .645 & .222 & .772 & .760 \\
\hline & 5 & .873 & .916 & .871 & .872 \\
\hline & 6 & .941 & .896 & .933 & .926 \\
\hline & 7 & .837 & .261 & .726 & .746 \\
\hline & 8 & .440 & .339 & .819 & .797 \\
\hline & 9 & .910 & .978 & .904 & .913 \\
\hline & 10 & .868 & .307 & .756 & .710 \\
\hline & 11 & .734 & .260 & .846 & .832 \\
\hline & 12 & .900 & .279 & .851 & .856 \\
\hline & 13 & .858 & .265 & .705 & .660 \\
\hline & Mean 1-13 & .818 & .461 & .827 & .811 \\
\hline & Whole pattern & .090 & .029 & .157 & .141 \\
\hline
\end{tabular}

Note-K-L, Kullback-Leibler. 
Table 10

Reading Test's Correct Attribute Mastery Classifications (Hit Rates) Using Fusion-Based Probabilities

\begin{tabular}{|c|c|c|c|c|c|}
\hline \multirow[b]{2}{*}{$Q$-Matrix } & \multirow[b]{2}{*}{ Attribute } & \multicolumn{2}{|c|}{$\begin{array}{l}\underline{\alpha} \text {-Based } \\
\text { Condition }\end{array}$} & \multicolumn{2}{|c|}{$\begin{array}{c}\theta \text { - and } \underline{\alpha} \text {-Based } \\
\text { Condition }\end{array}$} \\
\hline & & Shannon & $\mathrm{K}-\mathrm{L}$ & Shannon & $\mathrm{K}-\mathrm{L}$ \\
\hline \multirow[t]{8}{*}{ Blueprint } & 1 & .899 & .833 & .799 & .803 \\
\hline & 2 & .898 & .923 & .884 & .899 \\
\hline & 3 & .890 & .822 & .855 & .844 \\
\hline & 4 & .858 & .868 & .889 & .906 \\
\hline & 5 & .911 & .807 & .869 & .867 \\
\hline & 6 & .882 & .928 & .929 & .922 \\
\hline & Mean 1-6 & .890 & .863 & .871 & .874 \\
\hline & Whole pattern & .677 & .686 & .637 & .640 \\
\hline \multirow[t]{9}{*}{ Intuitive } & 1 & .929 & .967 & .924 & .941 \\
\hline & 2 & .882 & .881 & .883 & .887 \\
\hline & 3 & .888 & .898 & .896 & .889 \\
\hline & 4 & .908 & .866 & .880 & .859 \\
\hline & 5 & .900 & .867 & .867 & .868 \\
\hline & 6 & .919 & .891 & .901 & .896 \\
\hline & 7 & .893 & .862 & .893 & .885 \\
\hline & Mean 1-7 & .903 & .890 & .892 & .889 \\
\hline & Whole pattern & .711 & .722 & .699 & .708 \\
\hline
\end{tabular}

Note-K-L, Kullback-Leibler.

that the $\theta$ - and $\underline{\alpha}$-based condition performed better with regard to $\theta$ estimation than did the $\alpha$-based condition.) Within the $\theta$ - and $\alpha$-based condition, $\mathrm{K}$-L information and Shannon entropy seem to perform equally well in correctly estimating attribute mastery.

\section{Item Exposure}

In CAT, it is desirable to keep item exposure to a minimum to ensure test security. But some items are better at measuring the underlying construct than are others, so minimizing exposure control and maximizing measurement precision have a give-and-take relationship. Due to the importance of test security, the item exposure rates for the various methods implemented in this study were examined. The item exposure rates for the CAT simulation based on the 3PL model are presented in Table 11 for the math portion of the test and in Table 12 for the reading portion. Likewise, the item exposure rates for the CAT simulation based on the fusion model are presented in Table 13 for the math portion of the test and in Table 14 for the reading portion.

An example of desirable item exposure would involve all of the items exposed to fewer than $20 \%$ of the examinees and no items that were not administered at all. The 5-4-3-2-1 exposure control method did not perform as ideally as this, but the exposure tendencies can be compared across the various methods. For instance, the method in the $\underline{\alpha}$-based condition that maximized $\mathrm{K}-\mathrm{L}$ information in selecting the next item had between $2 \%$ and $6 \%$ of the items exposed to at least $90 \%$ of the examinees. This is an unacceptably high exposure rate. The $\theta$ - and $\underline{\alpha}$-based condition tended to have better exposure rates than did the $\underline{\alpha}$-based condition and tended to have exposure rates comparable to those in the $\theta$-based condition. The different methods within the $\theta$-based condition and within the $\theta$ - and $\alpha$-based condition also tended to be comparable with regard to exposure control. If these item exposure rates are greater than would be desired for a particular assessment, alternative exposure control techniques may be an interesting area of future research. Using a shadow test reduces item exposure by incorporating an ideal item exposure rate as a constraint in the integer programming algorithm.

\section{OVERALL PERFORMANCE}

Evaluation of the different techniques encompasses an evaluation of $\theta$ estimation accuracy, attribute mastery estimation accuracy, and item exposure control. Overall results should thus be considered across all three of these areas. With regard to estimating $\theta$, the $\theta$-based condition and the $\theta$ - and $\underline{\alpha}$-based condition produced comparable results, and performance in the $\theta$ - and $\underline{\alpha}$-based condition was better than that in the $\underline{\alpha}$-based condition. Surprisingly, the $\theta$-based condition and the $\theta$ - and $\underline{\alpha}$-based condition also produced comparable results with regard to the attribute mastery estimates. The $\theta$ - and $\underline{\alpha}$-based condition outperformed the $\underline{\alpha}$-based condition with regard to $\theta$ estimation, attribute mastery pattern estimation, and item exposure control. But between the $\theta$-based condition and the $\theta$ - and $\underline{\alpha}$-based condition, there was no clear-cut winner. Both methods performed well and similarly with regard to $\theta$ estimation, attribute mastery estimation, and item exposure control.

Note that the results for the intuitive $Q$-matrix for the reading test are quite poor with respect to all three of the discussed criteria and certainly show poorer performance than do the corresponding results based on the blueprint $Q$-matrix. This emphasizes the importance of $Q$-matrix construction. It would be inappropriate to underestimate the significance of $Q$-matrix development, so great care should go into this important initial step in the cognitive diagnosis process. Additional precautions could be taken, such as asking a content expert to review a $Q$-matrix before the cognitively diagnostic analysis or, depending on the resources available, asking multiple content experts to collaborate in the $Q$-matrix construction process.

In sum, both selecting items on the basis of current $\theta$ estimates alone or selecting items on the basis of both $\theta$ and attribute mastery estimates by means of the shadow test are good methods with regard to single-score estimation, attribute mastery estimation, and item exposure control. Consequently, choosing between these two methods must depend on other issues that a test administrator may be facing. If other constraints are necessary in the testing process, the shadow test approach that selects items on the basis of both single-score and attribute mastery estimation is better, because it can easily and efficiently incorporate the additional requirements. Such constraints include content balancing, item type constraints, and testlet constraints, among others. Van der Linden and Reese (1998) and van der Linden (2000) expound on incorporating such constraints.

On the other hand, if a test administrator prefers a more simple approach to item selection and does not have access to special software such as CPLEX (or the need for 


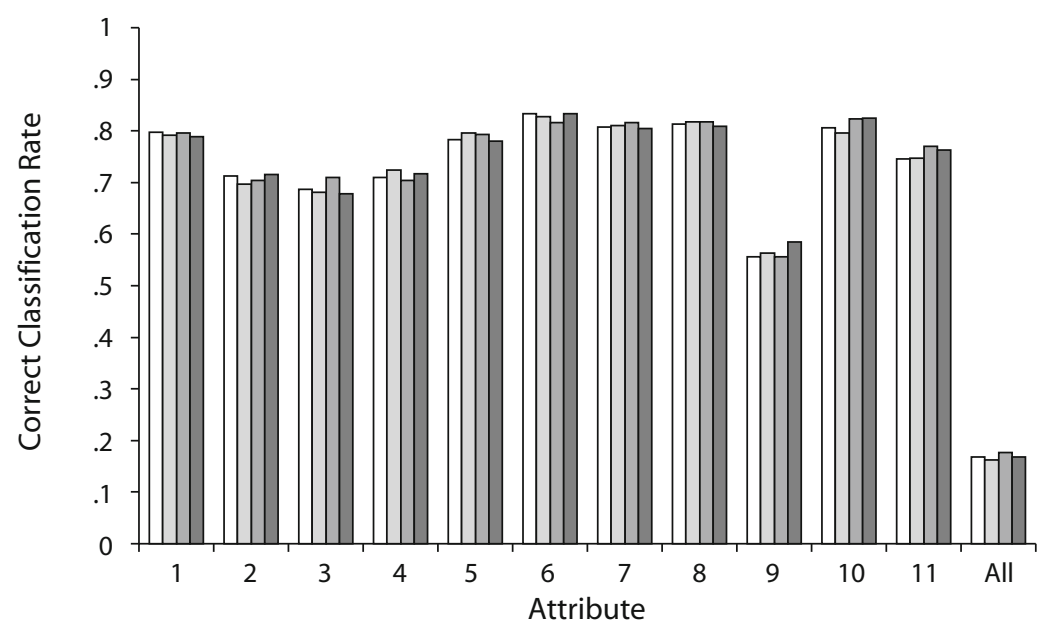

$\square$ Condition 1 Fischer $\square$ Condition 1 K-L $\quad \square$ Condition 3 K-L $\quad \square$ Condition 3 Shannon

Figure 1. Correct attribute mastery classification for the math blueprint $Q$-matrix using three-parameter-logistic (3PL)-based probabilities. K-L, Kullback-Leibler.

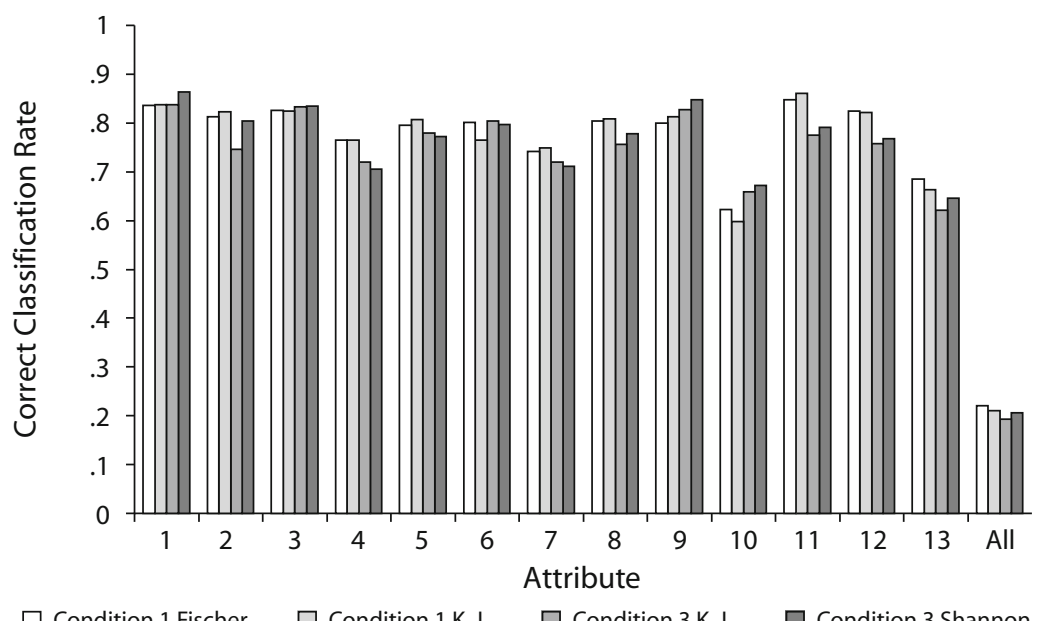

Figure 2. Correct attribute mastery classification for the math intuitive $Q$-matrix using 3PL-based probabilities. 


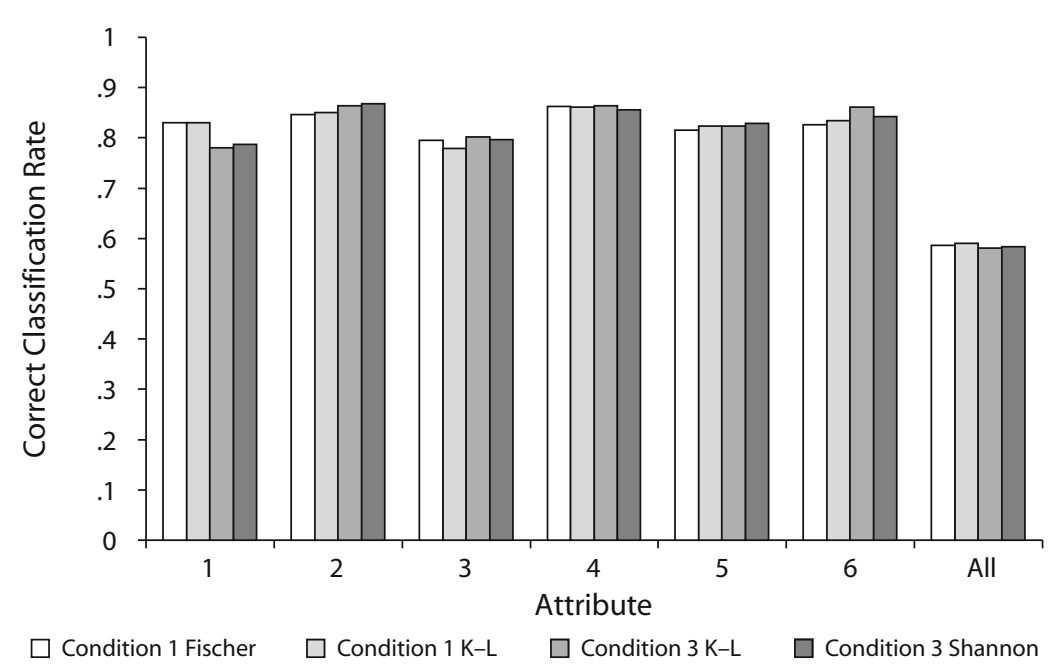

Figure 3. Correct attribute mastery classification for the reading blueprint $Q$-matrix using 3PL-based probabilities.

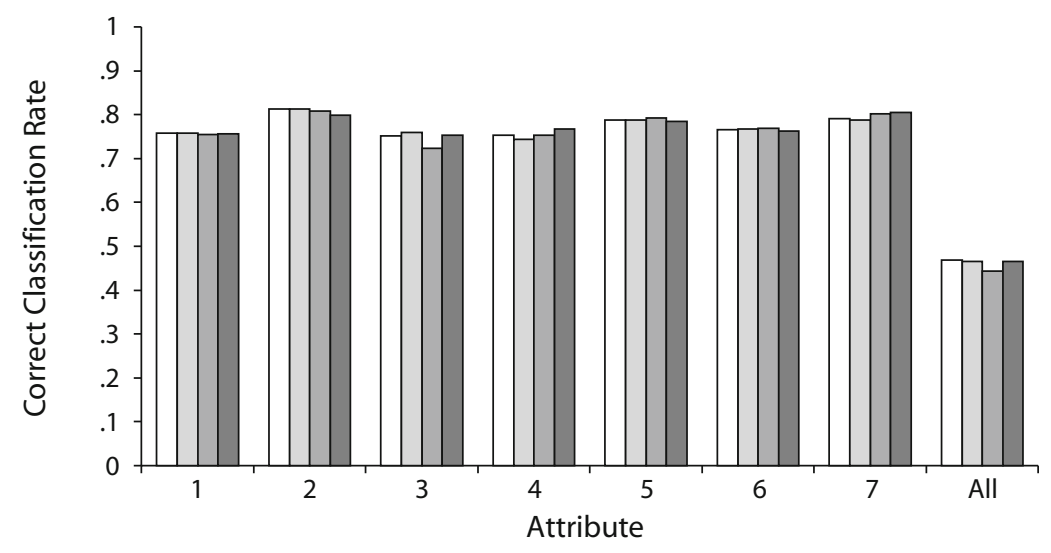

$\square$ Condition 1 Fischer $\quad \square$ Condition 1 K-L $\quad \square$ Condition 3 K-L $\quad \square$ Condition 3 Shannon

Figure 4. Correct attribute mastery classification for the reading intuitive $Q$-matrix using 3PL-based probabilities.

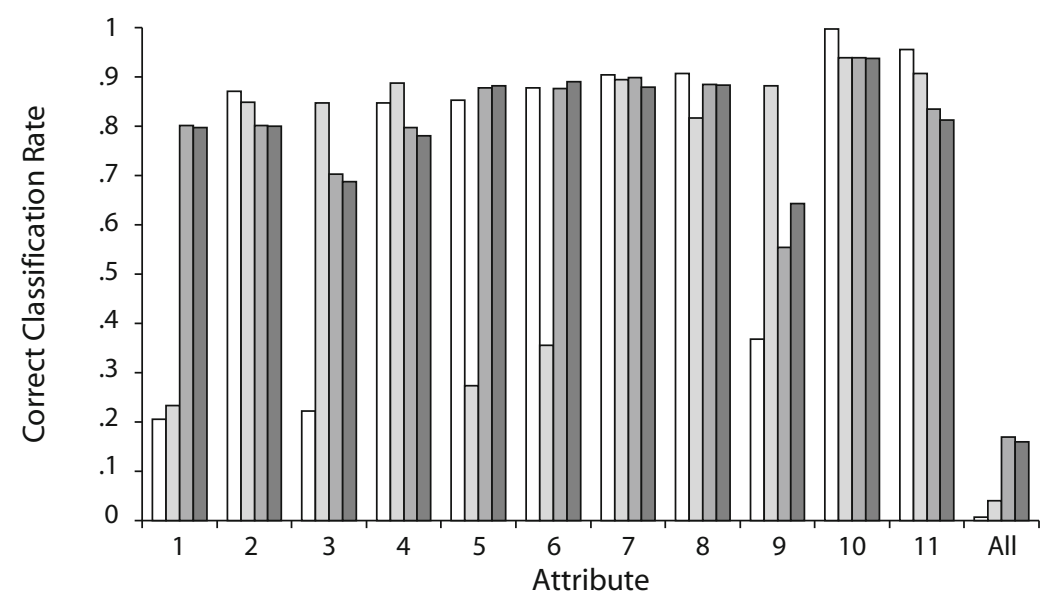

$\square$ Condition 2 K-L $\quad \square$ Condition 2 Shannon $\quad \square$ Condition 3 K-L $\quad \square$ Condition 3 Shannon

Figure 5. Correct attribute mastery classification for the math blueprint $Q$-matrix using fusion-based probabilities. 


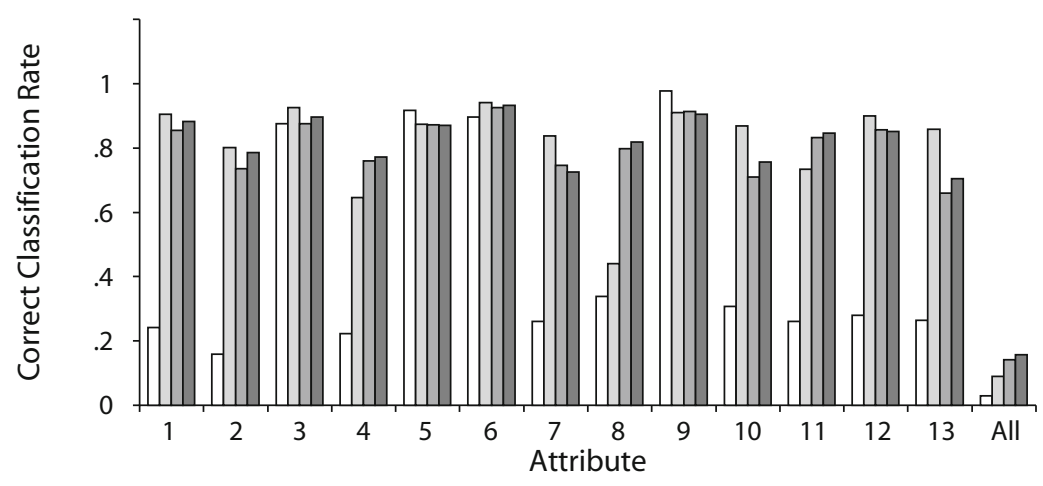

$\square$ Condition 2 K-L $\square$ Condition 2 Shannon $\square$ Condition 3 K-L $\quad \square$ Condition 3 Shannon

Figure 6. Correct attribute mastery classification for the math intuitive $Q$-matrix using fusion-based probabilities.

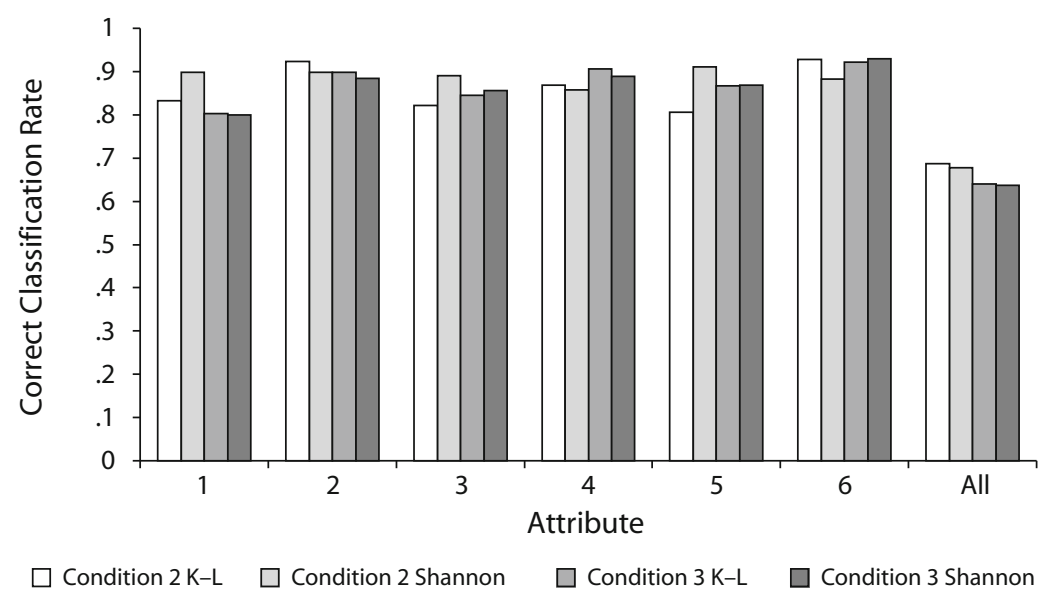

Figure 7. Correct attribute mastery classification for the reading blueprint $Q$-matrix using fusion-based probabilities.

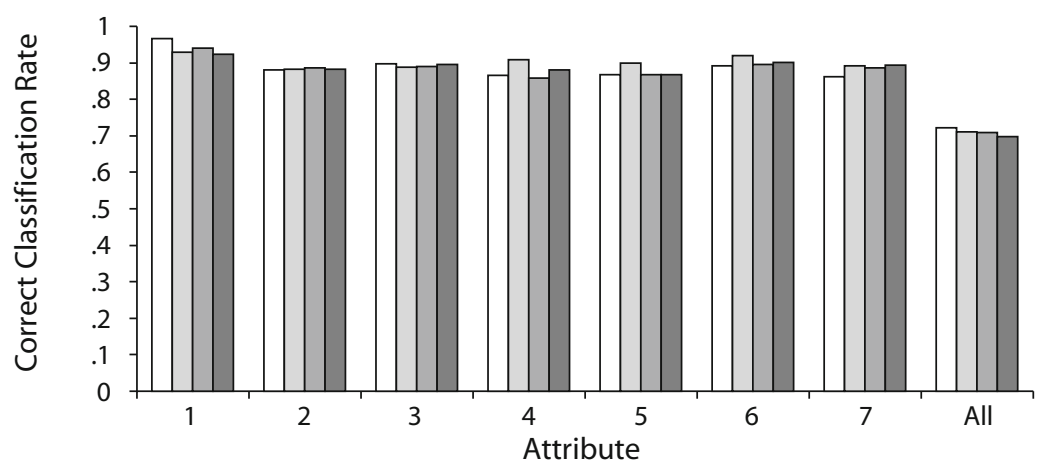

$\square$ Condition 2 K-L $\quad \square$ Condition 2 Shannon $\quad \square$ Condition 3 K-L $\quad \square$ Condition 3 Shannon

Figure 8. Correct attribute mastery classification for the reading intuitive $Q$-matrix using fusion-based probabilities. 
Table 11

Item Exposure for the Math Test Using

Three-Parameter-Logistic-Based Probabilities

\begin{tabular}{|c|c|c|c|c|c|c|c|c|}
\hline \multirow[b]{3}{*}{ Exposure Rate } & \multicolumn{4}{|c|}{$\theta$-Based Condition } & \multicolumn{4}{|c|}{$\theta$ - and $\underline{\alpha}$-Based Condition } \\
\hline & \multicolumn{2}{|c|}{ Fischer } & \multicolumn{2}{|c|}{$\mathrm{K}-\mathrm{L}$} & \multicolumn{2}{|c|}{ Shannon } & \multicolumn{2}{|c|}{$\mathrm{K}-\mathrm{L}$} \\
\hline & Freq. & Prop. & Freq. & Prop. & Freq. & Prop. & Freq. & Prop. \\
\hline \multicolumn{9}{|c|}{ Blueprint $Q$-Matrix } \\
\hline Not exposed & 114 & .29 & 153 & .39 & 137 & .35 & 147 & .37 \\
\hline $0-.099$ & 169 & .43 & 136 & .34 & 134 & .34 & 139 & .35 \\
\hline $.1-.199$ & 39 & .10 & 36 & .09 & 42 & .11 & 32 & .08 \\
\hline $.2-.299$ & 29 & .07 & 28 & .07 & 46 & .12 & 23 & .06 \\
\hline $.3-.399$ & 13 & .03 & 8 & .02 & 15 & .04 & 20 & .05 \\
\hline $.4-.499$ & 10 & .03 & 9 & .02 & 4 & .01 & 17 & .04 \\
\hline $.5-.599$ & 10 & .03 & 12 & .03 & 5 & .01 & 6 & .02 \\
\hline $.6-.699$ & 10 & .03 & 8 & .02 & 9 & .02 & 10 & .03 \\
\hline $.7-.799$ & 2 & .01 & 6 & .02 & 4 & .01 & 2 & .01 \\
\hline $.8-.899$ & 0 & 0 & 0 & 0 & 0 & 0 & 0 & 0 \\
\hline $.9-1.000$ & 0 & 0 & 0 & 0 & 0 & 0 & 0 & 0 \\
\hline \multicolumn{9}{|c|}{ Intuitive $Q$-Matrix } \\
\hline Not exposed & 152 & .38 & 170 & .43 & 126 & .32 & 122 & .31 \\
\hline $0-.099$ & 132 & .33 & 114 & .29 & 149 & .38 & 154 & .39 \\
\hline $.1-.199$ & 31 & .08 & 33 & .08 & 39 & .10 & 40 & .10 \\
\hline $.2-.299$ & 32 & .08 & 29 & .07 & 22 & .06 & 26 & .07 \\
\hline $.3-.399$ & 21 & .05 & 19 & .05 & 36 & .09 & 24 & .06 \\
\hline $.4-.499$ & 11 & .03 & 12 & .03 & 16 & .04 & 18 & .05 \\
\hline $.5-.599$ & 5 & .01 & 7 & .02 & 6 & .02 & 7 & .02 \\
\hline .6-.699 & 10 & .03 & 7 & .02 & 2 & .01 & 3 & .01 \\
\hline $.7-.799$ & 2 & .01 & 5 & .01 & 0 & 0 & 2 & .01 \\
\hline $.8-.899$ & 0 & 0 & 0 & 0 & 0 & 0 & 0 & 0 \\
\hline $.9-1.000$ & 0 & 0 & 0 & 0 & 0 & 0 & 0 & 0 \\
\hline
\end{tabular}

Note-Freq., frequency; Prop., proportion; K-L, Kullback-Leibler.

Table 12

Item Exposure for the Reading Test Using Three-Parameter-Logistic-Based Probabilities

\begin{tabular}{|c|c|c|c|c|c|c|c|c|}
\hline \multirow[b]{3}{*}{ Exposure Rate } & \multicolumn{4}{|c|}{$\theta$-Based Condition } & \multicolumn{4}{|c|}{$\theta$ - and $\underline{\alpha}$-Based Condition } \\
\hline & \multicolumn{2}{|c|}{ Fischer } & \multicolumn{2}{|c|}{$\mathrm{K}-\mathrm{L}$} & \multicolumn{2}{|c|}{ Shannon } & \multicolumn{2}{|c|}{$\mathrm{K}-\mathrm{L}$} \\
\hline & Freq. & Prop. & Freq. & Prop. & Freq. & Prop. & Freq. & Prop. \\
\hline \multicolumn{9}{|c|}{ Blueprint $Q$-Matrix } \\
\hline Not exposed & 100 & .25 & 116 & .29 & 121 & .31 & 123 & .31 \\
\hline $0-.099$ & 115 & .29 & 100 & .25 & 115 & .29 & 103 & .26 \\
\hline $.1-.199$ & 32 & .08 & 37 & .09 & 22 & .06 & 34 & .09 \\
\hline $.2-.299$ & 28 & .07 & 21 & .05 & 12 & .03 & 12 & .03 \\
\hline $.3-.399$ & 13 & .03 & 17 & .04 & 13 & .03 & 17 & .04 \\
\hline $.4-.499$ & 14 & .04 & 9 & .02 & 18 & .05 & 5 & .01 \\
\hline $.5-.599$ & 11 & .03 & 8 & .02 & 6 & .02 & 9 & .02 \\
\hline $.6-.699$ & 8 & .02 & 9 & .02 & 6 & .02 & 13 & .03 \\
\hline $.7-.799$ & 0 & 0 & 4 & .01 & 5 & .01 & 5 & .01 \\
\hline $.8-.899$ & 3 & .01 & 2 & .01 & 6 & .02 & 3 & .01 \\
\hline $.9-1.000$ & 0 & 0 & 1 & 0 & 0 & 0 & 0 & 0 \\
\hline \multicolumn{9}{|c|}{ Intuitive $Q$-Matrix } \\
\hline Not exposed & 100 & .25 & 111 & .28 & 103 & .26 & 109 & .28 \\
\hline $0-.099$ & 115 & .29 & 106 & .27 & 115 & .29 & 114 & .29 \\
\hline $.1-.199$ & 32 & .08 & 36 & .09 & 32 & .08 & 30 & .08 \\
\hline $.2-.299$ & 26 & .07 & 22 & .06 & 16 & .04 & 13 & .03 \\
\hline $.3-.399$ & 8 & .02 & 13 & .03 & 14 & .04 & 19 & .05 \\
\hline $.4-.499$ & 20 & .05 & 11 & .03 & 22 & .06 & 13 & .03 \\
\hline $.5-.599$ & 10 & .03 & 10 & .03 & 15 & .04 & 13 & .03 \\
\hline $.6-.699$ & 8 & .02 & 6 & .02 & 1 & 0 & 10 & .03 \\
\hline $.7-.799$ & 3 & .01 & 7 & .02 & 6 & .02 & 3 & .01 \\
\hline $.8-.899$ & 0 & 0 & 2 & .01 & 0 & 0 & 0 & 0 \\
\hline $.9-1.000$ & 0 & 0 & 0 & 0 & 0 & 0 & 0 & 0 \\
\hline
\end{tabular}

Note-Freq., frequency; Prop., proportion; K-L, Kullback-Leibler. 
Table 13

Item Exposure for the Math Test Using Fusion-Based Probabilities

\begin{tabular}{|c|c|c|c|c|c|c|c|c|}
\hline \multirow[b]{3}{*}{ Exposure Rate } & \multicolumn{4}{|c|}{$\underline{\alpha}$-Based Condition } & \multicolumn{4}{|c|}{$\theta$ - and $\underline{\alpha}$-Based Condition } \\
\hline & \multicolumn{2}{|c|}{ Shannon } & \multicolumn{2}{|c|}{$\mathrm{K}-\mathrm{L}$} & \multicolumn{2}{|c|}{ Shannon } & \multicolumn{2}{|c|}{$\mathrm{K}-\mathrm{L}$} \\
\hline & Freq. & Prop. & Freq. & Prop. & Freq. & Prop. & Freq. & Prop. \\
\hline \multicolumn{9}{|c|}{ Blueprint $Q$-Matrix } \\
\hline Not exposed & 156 & .39 & 311 & .79 & 160 & .40 & 181 & .46 \\
\hline $0-.099$ & 134 & .34 & 22 & .06 & 116 & .29 & 108 & .27 \\
\hline $.1-.199$ & 42 & .11 & 15 & .04 & 51 & .13 & 34 & .09 \\
\hline $.2-.299$ & 11 & .03 & 8 & .02 & 29 & .07 & 19 & .05 \\
\hline $.3-.399$ & 13 & .03 & 0 & 0 & 8 & .02 & 17 & .04 \\
\hline $.4-.499$ & 11 & .03 & 0 & 0 & 12 & .03 & 7 & .02 \\
\hline $.5-.599$ & 13 & .03 & 2 & .01 & 3 & .01 & 17 & .04 \\
\hline $.6-.699$ & 9 & .02 & 3 & .01 & 9 & .02 & 3 & .01 \\
\hline $.7-.799$ & 5 & .01 & 8 & .02 & 7 & .02 & 8 & .02 \\
\hline $.8-.899$ & 1 & 0 & 6 & .02 & 1 & 0 & 2 & .01 \\
\hline $.9-1.000$ & 1 & 0 & 21 & .05 & 0 & 0 & 0 & 0 \\
\hline \multicolumn{9}{|c|}{ Intuitive $Q$-Matrix } \\
\hline Not exposed & 127 & .32 & 205 & .52 & 139 & .35 & 148 & .37 \\
\hline $0-.099$ & 145 & .37 & 115 & .29 & 143 & .36 & 130 & .33 \\
\hline $.1-.199$ & 50 & .13 & 21 & .05 & 43 & .11 & 44 & .11 \\
\hline $.2-.299$ & 28 & .07 & 13 & .03 & 19 & .05 & 18 & .05 \\
\hline $.3-.399$ & 21 & .05 & 2 & .01 & 19 & .05 & 28 & .07 \\
\hline $.4-.499$ & 17 & .04 & 0 & 0 & 19 & .05 & 9 & .02 \\
\hline $.5-.599$ & 5 & .01 & 5 & .01 & 5 & .01 & 7 & .02 \\
\hline $.6-.699$ & 1 & 0 & 9 & .02 & 5 & .01 & 6 & .02 \\
\hline $.7-.799$ & 1 & 0 & 16 & .04 & 4 & .01 & 5 & .01 \\
\hline $.8-.899$ & 1 & 0 & 2 & .01 & 0 & 0 & 1 & 0 \\
\hline $.9-1.000$ & 0 & 0 & 8 & .02 & 0 & 0 & 0 & 0 \\
\hline
\end{tabular}

Note-Freq., frequency; Prop., proportion; K-L, Kullback-Leibler.

Table 14

Item Exposure for the Reading Test Using Fusion-Based Probabilities

\begin{tabular}{|c|c|c|c|c|c|c|c|c|}
\hline \multirow[b]{3}{*}{ Exposure Rate } & \multicolumn{4}{|c|}{$\underline{\alpha}$-Based Condition } & \multicolumn{4}{|c|}{$\theta$ - and $\underline{\alpha}$-Based Condition } \\
\hline & \multicolumn{2}{|c|}{ Shannon } & \multicolumn{2}{|c|}{$\mathrm{K}-\mathrm{L}$} & \multicolumn{2}{|c|}{ Shannon } & \multicolumn{2}{|c|}{$\mathrm{K}-\mathrm{L}$} \\
\hline & Freq. & Prop. & Freq. & Prop. & Freq. & Prop. & Freq. & Prop. \\
\hline \multicolumn{9}{|c|}{ Blueprint $Q$-Matrix } \\
\hline Not exposed & 93 & .23 & 200 & .51 & 131 & .33 & 134 & .34 \\
\hline $0-.099$ & 131 & .33 & 71 & .18 & 95 & .24 & 101 & .26 \\
\hline $.1-.199$ & 51 & .13 & 7 & .02 & 35 & .09 & 25 & .06 \\
\hline $.2-.299$ & 7 & .02 & 4 & .01 & 10 & .03 & 20 & .05 \\
\hline $.3-.399$ & 2 & .01 & 1 & 0 & 11 & .03 & 3 & .01 \\
\hline $.4-.499$ & 7 & .02 & 1 & 0 & 13 & .03 & 8 & .02 \\
\hline $.5-.599$ & 7 & .02 & 2 & .01 & 10 & .03 & 2 & .01 \\
\hline $.6-.699$ & 13 & .03 & 2 & .01 & 3 & .01 & 9 & .02 \\
\hline $.7-.799$ & 9 & .02 & 6 & .02 & 9 & .02 & 17 & .04 \\
\hline $.8-.899$ & 3 & .01 & 7 & .02 & 7 & .02 & 2 & .01 \\
\hline $.9-1.000$ & 1 & 0 & 23 & .06 & 0 & 0 & 3 & .01 \\
\hline \multicolumn{9}{|c|}{ Intuitive $Q$-Matrix } \\
\hline Not exposed & 87 & .22 & 194 & .49 & 102 & .26 & 114 & .29 \\
\hline $0-.099$ & 135 & .34 & 81 & .20 & 112 & .28 & 117 & .30 \\
\hline $.1-.199$ & 48 & .12 & 7 & .02 & 44 & .11 & 31 & .08 \\
\hline $.2-.299$ & 12 & .03 & 2 & .01 & 9 & .02 & 10 & .03 \\
\hline $.3-.399$ & 6 & .02 & 0 & 0 & 16 & .04 & 7 & .02 \\
\hline $.4-.499$ & 2 & .01 & 0 & 0 & 18 & .05 & 7 & .02 \\
\hline $.5-.599$ & 10 & .03 & 0 & 0 & 15 & .04 & 17 & .04 \\
\hline $.6-.699$ & 9 & .02 & 2 & .01 & 2 & .01 & 13 & .03 \\
\hline $.7-.799$ & 13 & .03 & 11 & .03 & 6 & .02 & 7 & .02 \\
\hline $.8-.899$ & 2 & .01 & 2 & .01 & 0 & 0 & 1 & 0 \\
\hline $.9-1.000$ & 0 & 0 & 25 & .06 & 0 & 0 & 0 & 0 \\
\hline
\end{tabular}

Note-Freq., frequency; Prop., proportion; K-L, Kullback-Leibler. 
additional constraints), an item selection method based on the current single-score estimates will suffice. Furthermore, the different approaches within these item selection techniques (i.e., Fischer information vs. $\mathrm{K}-\mathrm{L}$ information and Shannon entropy vs. K-L information) seem to have made little difference in the results of this study, so a test administrator may have his or her choice between these.

Because the shadow test approach that selects items on the basis of both single-score and attribute mastery estimation performs comparably to the method based on the single-score estimate when the 3PL model is used for generating item response probabilities, this new method can be used immediately in accordance with existing testing methods. This is a benefit that is not shared by all cognitively diagnostic assessment methods. This means that this new method of using the shadow test to select items on the basis of both the current single-score and attribute mastery estimates can immediately provide the benefits of cognitively diagnostic assessment in existing testing programs.

\section{FUTURE DIRECTIONS OF RESEARCH}

This study compared the accuracy of three possible item selection methods in a CAT situation focused on estimating diagnostic attribute information in addition to the conventional single-score estimation. This simulation study is important in an educational context because it explores the accuracy of these methods with regard to these assessment approaches. Test administrators can also use simulation studies such as this one to determine how well the various attributes of interest are being measured. For instance, in Figures 1 and 2, the mastery status for Attributes 9 and 10, respectively, are not estimated as accurately as the other attributes. Note that both of these attributes deal with the students' ability to estimate a reasonable solution to the item. Test administrators can use this information to evaluate poorly measured attributes or to examine items measuring an attribute to try to better assess what they originally had in mind for this attribute. The results of this study also stress the importance of the construction of the $Q$-matrix in cognitively diagnostic assessment.

Cognitive diagnosis is important in educational assessment because it provides helpful feedback to students about specific elements of the measured content domain. It is rapidly becoming a requirement of effective, educationally beneficial test development (No Child Left Behind Act; U.S. House of Representatives, 2001). The challenge then becomes how to adapt the methods developed within the CAT framework to enable this new approach. This study proposes the application of the shadow test pro- cedure to achieve the best of both worlds. Although this study was conducted using the fusion model's framework for cognitive diagnosis, the procedure can be generalized to any diagnostic model that estimates the attribute states of the examinees, such as the noisy inputs deterministic "and" gate model (see Maris, 1999), the generalized latent trait model (Embretson, 1984), or the rule space method (Tatsuoka \& Tatsuoka, 1982).

\section{AUTHOR NOTE}

Correspondence concerning this article should be addressed to M. McGlohen, Division of Instructional Innovation and Assessment, University of Texas, P.O. Box 7246, 2616 Wichita St., Mail Code E3000, Austin, TX 78713 (e-mail: meghan.mcglohen@austin.utexas.edu).

\section{REFERENCES}

Chang, H.-H., \& YING, Z. (1999). $\alpha$-Stratified multistage computerized adaptive testing. Applied Psychological Measurement, 23, 211-222.

Chipman, S. F., Nichols, P. D., \& Brennan, R. L. (1995). Introduction. In P. D. Nichols, S. F. Chipman, \& R. L. Brennan (Eds.), Cognitively diagnostic assessment (pp. 1-18). Hillsdale, NJ: Erlbaum.

DiBello, L. V., Stout, W. F., \& Roussos, L. A. (1995). Unified cognitive/psychometric diagnostic assessment likelihood-based classification techniques. In P. D. Nichols, S. F. Chipman, \& R. L. Brennan (Eds.), Cognitively diagnostic assessment (pp. 361-389). Hillsdale, NJ: Erlbaum.

Educational Testing Service (2004). Arpeggio: Release 1.1 [Computer software]. Princeton, NJ: Author.

Embretson, S. (1984). A general latent trait model for response processes. Psychometrika, 49, 175-186.

Fischer, G. H. (1973). The linear logistic test model as an instrument in educational research. Acta Psychologica, 37, 359-374.

Hartz, S., Roussos, L., \& Stout, W. (2002). Skills diagnosis: Theory and practice [User manual for Arpeggio software]. Princeton, NJ: Educational Testing Service.

MARIs, E. (1999). Estimating multiple classification latent class models. Psychometrika, 64, 187-212.

MCBRIDE, J. R., \& MARTIN, J. T. (1983). Reliability and validity of adaptive ability tests in a military setting. In D. J. Weiss (Ed.), New horizons in testing (pp. 223-236). New York: Academic Press.

Shannon, C. E. (1948). A mathematical theory of communication. Bell System Technical Journal, 27, 379-423, 623-656.

TAtsuoka, K. K., \& TAтsuokA, M. M. (1982). Detection of aberrant response patterns and their effect on dimensionality. Journal of Educational Statistics, 7, 215-231.

U.S. House of Representatives (2001). No Child Left Behind Act, Public Law 107-110, 115 Stat. 1425.

VAN DER LINDEN, W. J. (2000). Constrained adaptive testing with shadow tests. In W. J. van der Linden \& C. A. W. Glas (Eds.), Computerized adaptive testing: Theory and practice (pp. 27-52). Dordrecht: Kluwer.

VAN DER LINDEN, W. J., \& CHANG, H.-H. (2003). Implementing content constraints in alpha-stratified adaptive testing using a shadow test approach. Applied Psychological Measurement, 27, 107-120.

VAN DER Linden, W. J., \& Reese, L. M. (1998). A model for optimal constrained adaptive testing. Applied Psychological Measurement, 22, 259-270.

Xu, X., Chang, H.-H., \& Douglas, J. (2003, April). A simulation study to compare CAT strategies for cognitive diagnosis. Paper presented at the annual meeting of the American Educational Research Association, Chicago. 


\section{APPENDIX}

List of Attributes Measured by Each Test

\section{Blueprint $Q$-Matrix for the Math Test}

1. Demonstrate an understanding of number concepts.

2. Demonstrate an understanding of mathematical relations.

3. Demonstrate an understanding of geometric properties and relationships.

4. Demonstrate an understanding of measurement concepts using metric and customary units.

5. Demonstrate an understanding of probability and statistics.

6. Use the operation of addition to solve problems.

7. Use the operation of subtraction to solve problems.

8. Use the operation of multiplication and/or division to solve problems.

9. Estimate solutions to a problem situation and/or evaluate the reasonableness of a solution to a problem situation.

10. Determine solution strategies and analyze or solve problems.

11. Express or solve problems using mathematical representation.

\section{Intuitive $Q$-Matrix of the Math Test}

1. Understanding representation

2. Counting

3. Multiplication

4. Division

5. Addition

6. Subtraction

7. Understanding geometric shapes (turning, flipping, draw a line of symmetry, etc.)

8. Read a chart

9. Set up an arithmetic calculation from verbal information

10. Estimation

11. Read a table of numbers

12. Using standard units of measure

13. Understanding and forming order of magnitude

\section{Blueprint $Q$-Matrix for the Reading Test}

1. Determine the meaning of words in a variety of written texts.

2. Identify supporting ideas in a variety of written texts.

3. Summarize a variety of written texts.

4. Perceive relationships and recognize outcomes in a variety of written texts.

5. Analyze information in a variety of written texts in order to make inferences and generalizations.

6. Recognize points of view, propaganda, and/or statements of fact and opinion in a variety of written texts.

\section{Intuitive $Q$-Matrix of the Reading Test}

1. Chronology

2. Causality (determining why)

3 . Word meaning

4. General summary

5. Observing/remembering details

6. Knowing fact versus opinion

7. Speculating from contextual clues 